\title{
IACUC Replacement Parts: What are the Requirements?
}

Dr. Billy Williams was President and CEO of Advanced Medical Dynamics (AMD), a small but very successful company that developed disposable medical devices. Because AMD had small-business grants from the NIH, the company had filed an Assurance with OLAW. Williams was listed on the Assurance as both the CEO who appointed the members of the IACUC and the Institutional Official (IO).

Like the company itself, the AMD IACUC was small but effective. It consisted of a Chair, Dr. Dawn Connors, who was a scientist at AMD, and another AMD scientist who, like Connors, was a user of animals. There were also the AMD business manager, the consulting veterinarian, and the Reverend Harrison Bailey, a local pastor who had no affiliation with the company. The IACUC typically met every 3 months.

A unique sequence of events created a problem for this small IACUC. Research activity came together in such a way that, a few days before the meeting, Connors found that, in addition to the usual business, there was one 'high-priority' protocol for the NIH work that, for all practical purposes, could not be delayed. Under normal circumstances that pressure would have been little more than an inconvenience, but on the same day Bailey abruptly resigned from the Committee, citing pressing personal reasons. Connors asked the pastor to consider sending a letter of resignation that would become effective after the next meeting, even if Bailey would not be attending, or even the next day, so she could quickly arrange for a Designated Member review. This suggestion seemed unethical to Bailey, and he politely declined to do so. The situation went from bad to worse when Connors recalled that Williams and his wife were on a 25th wedding anniversary trip down the Amazon and would not be reach- able for almost a month. Williams had designated Jack Friedman to make major business decisions in his absence and made that known to everybody in the company.

Connors was uncertain, but assumed that she could not have a meeting without the unaffiliated member. She went to Friedman and told him that they would have to replace Bailey quickly. "No problem at all," said Friedman, "I'll get my next door neighbor to fill in for the meeting. He's a retired attorney with no affiliation to us, and he knows everybody in town. He's the guy who represented us when we were thinking of merging with Xeon. I think that's how he made enough money to retire, so he owes me a favor." "Thanks," said Connors, "if that works it should solve things until Billy gets back and can appoint a permanent member, although, to tell you the truth Jack, I'm not sure if you're allowed to appoint anyone." "Look," said Friedman, "Don't worry. Billy gave me the authority to make decisions in his absence, and that's what I'm doing. But even if it turns out that it's not entirely kosher, so what? It's only one meeting and it fulfills the spirit of the NIH regs. Go home and don't lose any sleep over this. There's no harm done." "Yeah, you're right," said Connors, with a half-smile creeping over her face. "No harm done."

Were the actions taken by Friedman and Connors on behalf of AMD appropriate from both the regulatory and ethical perspectives? Would you have done otherwise?

\section{No Authority}

\section{Ellen M. Levee, DVM}

It is imperative for IACUCs to maintain properly constituted membership in accordance with federal regulations. The authority to appoint this membership lies with the IO or CEO. The Health Research Act of 1985 requires that the CEO of the entity for which the IACUC is established appoint the Committee ${ }^{1}$. According to the PHS Policy on Humane Care and Use of Laboratory Animals (Policy), "OLAW considers the CEO to be the highest operating official of the organization. If the CEO delegates authority to appoint the IACUC then the delegation must be specific and in writing ${ }^{2 "}$.

AMD's situation seems to lie outside of this requirement. Although Williams may have designated Friedman to make major business decisions in his absence, he obviously did not specifically delegate to Friedman the appointment of IACUC members. Furthermore, there seems to be no documentation to support Friedman's authority as the acting IO. As anyone in the animal care field knows, if it is not documented it doesn't count! Therefore, Friedman cannot appoint his neighbor as the unaffiliated member.

The objective of the PHS Policy with regard to the unaffiliated member is that the person "is not affiliated with the institution in any way other than as a member of the IACUC ${ }^{3}$." Because Friedman's neighbor previously represented AMD as an attorney, he clearly does not meet the intent of the PHS Policy with regard to affiliation. The unaffiliated member of the IACUC plays an extremely important role in the oversight process. Because the unaffiliated member is supposed to represent the general community's interests and concerns, it certainly might cause some consternation among everyone concerned that this person had previously made a considerable fortune representing AMD. The credibility of the company to fulfill the intent of the regulations with 